Provided for non-commercial research and educational use only. Not for reproduction or distribution or commercial use.
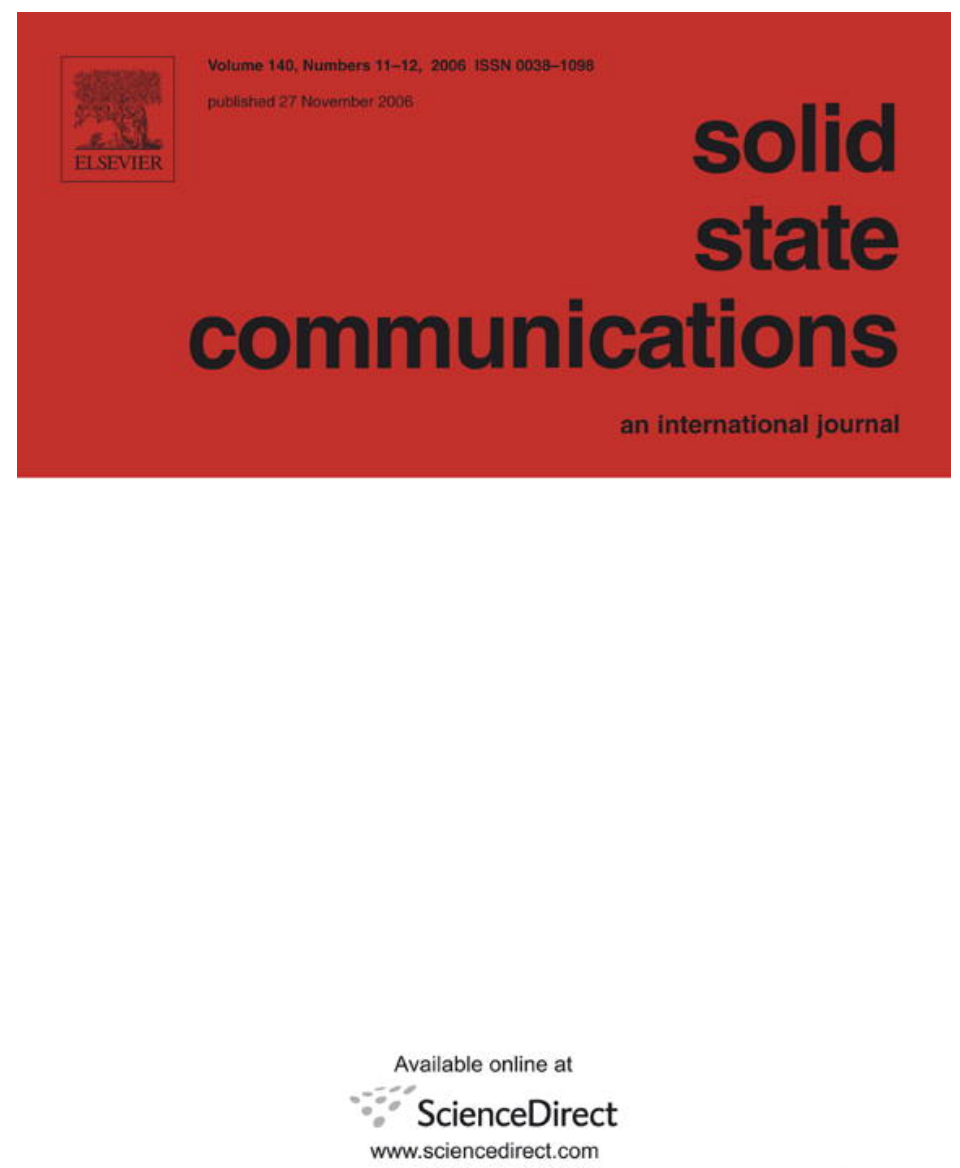

www.elsevier.com/locate/ssc

This article was originally published in a journal published by Elsevier, and the attached copy is provided by Elsevier for the author's benefit and for the benefit of the author's institution, for non-commercial research and educational use including without limitation use in instruction at your institution, sending it to specific colleagues that you know, and providing a copy to your institution's administrator.

All other uses, reproduction and distribution, including without limitation commercial reprints, selling or licensing copies or access,

or posting on open internet sites, your personal or institution's website or repository, are prohibited. For exceptions, permission may be sought for such use through Elsevier's permissions site at:

http://www.elsevier.com/locate/permissionusematerial 


\title{
Analysis of thermionic emission from electrodeposited Ni-Si Schottky barriers
}

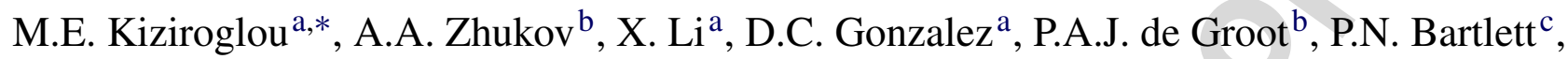 \\ C.H. de Groot ${ }^{\mathrm{a}}$ \\ ${ }^{a}$ School of Electronics and Computer Science, University of Southampton, SO17 1BJ, Southampton, UK \\ ${ }^{\mathrm{b}}$ School of Physics and Astronomy, University of Southampton, SO17 1BJ, Southampton, UK \\ ${ }^{\mathrm{c}}$ School of Chemistry, University of Southampton, SO17 1BJ, Southampton, UK
}

Received 2 May 2006; received in revised form 14 September 2006; accepted 15 September 2006 by J.A. Brum

Available online 28 September 2006

\begin{abstract}
$\mathrm{Ni}-\mathrm{Si}$ Schottky barriers are fabricated by electrodeposition using $n$ on $n+\mathrm{Si}$ substrates. $I-V, C-V$ and low temperature $I-V$ measurements are presented. A high-quality Schottky barrier with extremely low reverse leakage current is revealed. The results are shown to fit an inhomogeneous barrier model for thermionic emission over a Schottky barrier proposed by Werner and Guttler [J.H. Werner, H.H. Guttler, Barrier inhomogeneities at Schottky contacts, J. Appl. Phys. 69 (3) (1991) 1522-1533]. A mean value of $0.76 \mathrm{~V}$ and a standard deviation of $66 \mathrm{mV}$ is obtained for the Schottky barrier height at room temperature with a linear bias dependence. X-ray diffraction and scanning electron microscopy measurements reveal a polycrystalline Ni film with grains that span from the Ni-Si interface to the top of the Ni layer. The variation in Ni orientation is suggested as a possible source of the spatial distribution of the Schottky barrier height.
\end{abstract}

(c) 2006 Elsevier Ltd. All rights reserved.

PACS: $73.30 .+\mathrm{y}$

Keywords: A. Magnetic materials; B. Electrodeposition; D. Schottky barriers

\section{Introduction}

Spin injection from a magnetic metal into a semiconductor requires spin conservation during the injection of the electrons. It is particularly interesting because it allows the integration of magnetic devices with microelectronics. The conductivity mismatch problem has been shown to prevent ohmic contacts from being used for spin injection [1,2]. Instead, Schottky Barriers (SBs) and Ferromagnet (FM)/Insulator/Semiconductor contacts have been used [3]. An FM/Si/FM spintronic device has been proposed by Bratkovsky and Osipov using SBs for injection and extraction of spin polarized electrons [4]. A detailed understanding of the transport

\footnotetext{
* Corresponding address: School of Electronics and Computer Science, University of Southampton, Highfield Campus, SO171BJ, Southampton, Hampshire, UK. Tel.: +44 238055 2265; fax: +44 2380593029.

E-mail address: kmike@ soton.ac.uk (M.E. Kiziroglou).
}

mechanisms in SBs is required for the fabrication of such devices.

In a previous paper, we have shown that electrodeposition is a promising technique for the fabrication of $\mathrm{Ni}-\mathrm{Si} \mathrm{SBs}$ [5]. Here, the detailed characterization of electrodeposited $\mathrm{Ni}-\mathrm{Si}$ $\mathrm{SBs}$ is presented. $\mathrm{N}$ on $n+\mathrm{Si}$ substrates are used. The $n \mathrm{Si}$ layer is used to eliminate tunneling effects and allow for a detailed characterization of the SB by applying the thermionic emission theory. The $n+$ bulk is used to minimize the series resistance and increase the exponential region at the forward bias. Room temperature $I-V$ measurements are compared with those obtained from evaporated $\mathrm{Ni}-\mathrm{Si} \mathrm{SBs}$, and with corresponding results from the literature. $C-V$ and low temperature $I-V$ measurements are presented and interpreted according to the thermionic emission theory. The temperature dependence of the SB height is analyzed using recent theoretical models. Furthermore, a possible physical explanation of the spatial SB distribution is given using X-ray diffraction and Scanning Electron Microscopy measurements. 


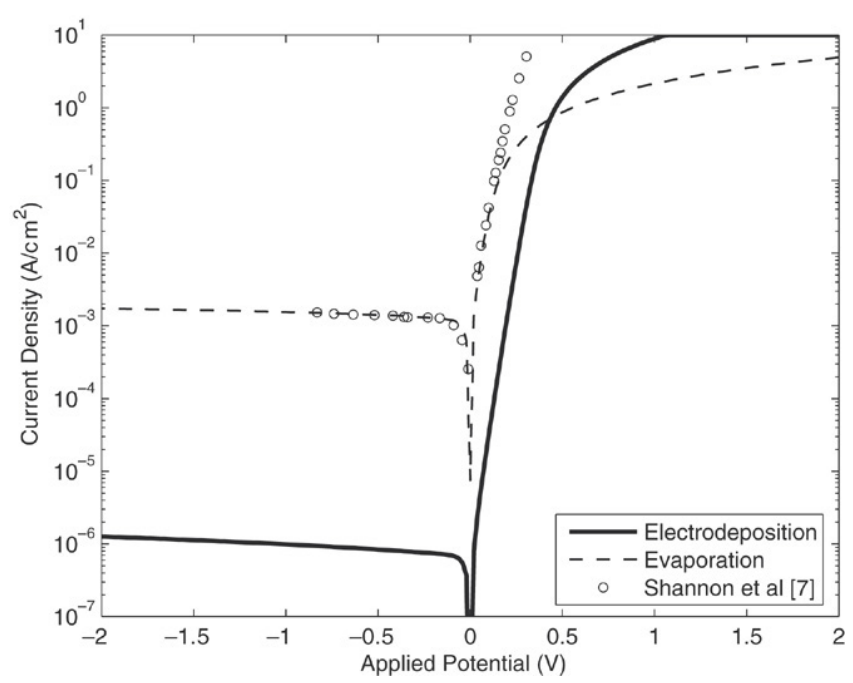

Fig. 1. $J-V$ characteristics of electrodeposited and evaporated Ni-Si SBs. $\mathrm{N}$ on $n+\mathrm{Si}$ with resistivity $0.9-1 \Omega \mathrm{cm} / 0.01-0.02 \Omega \mathrm{cm}$ was used for the electrodeposited SBs while 10-20 $\Omega \mathrm{cm}$ Si was used for the evaporated ones. Corresponding results from the literature for evaporated $\mathrm{Ni}-\mathrm{Si} \mathrm{SBs}$ are also shown for reference.

\section{Experimental procedure}

For the fabrication of $\mathrm{Ni}-\mathrm{Si} \mathrm{SBs}$, epitaxial $n$ on $n+\langle 100\rangle$ single-side polished silicon wafers with resistivity $0.9-1 \Omega \mathrm{cm} / 0.01-0.02 \Omega \mathrm{cm}$ were used as substrates. The thickness of the $n$ layer was $6-8 \mu \mathrm{m}$. A $20 \mathrm{~nm}$ thick layer of $\mathrm{SiO}_{2}$ was thermally grown on the front side. Circular and square patterns of sizes from $0.1 \mathrm{~mm}$ to $1.5 \mathrm{~mm}$ were transferred to the $\mathrm{SiO}_{2}$ layer by conventional lithography. $\mathrm{Al}$ ohmic back contacts were created by evaporation and annealing for $30 \mathrm{~min}$ at $450{ }^{\circ} \mathrm{C}$ in $\mathrm{H}_{2} / \mathrm{N}_{2}$ to allow for electrical measurements. Subsequently, after a standard RCA cleaning step (Radio Corporation of America, $\mathrm{H}_{2} \mathrm{O}_{2} / \mathrm{NH}_{4} \mathrm{OH}$ followed by $\mathrm{H}_{2} \mathrm{O}_{2} / \mathrm{HCl}$ ) and a $20: 1$ buffered HF dip for $30 \mathrm{~s}$, electrodeposition of $\mathrm{Ni}$ directly on $\mathrm{Si}$ was performed. The role of the HF dip is essential for the fabrication process as it removes the native oxide and prevents its reformation by leaving the $\mathrm{Si}$ surface H-terminated [6]. Moreover, the saturation of the dangling bonds on the Si surface by $\mathrm{H}$-termination reduces the formation of interface defects during Ni deposition [7].

For electrodeposition, a Ni sulphate bath and an Autolab AUT72032 potentiostat three-electrode system with a Pt counter electrode and a saturated calomel reference electrode (SCE) were used [8]. The deposition potential was $-1.1 \mathrm{~V}$ (against the SCE). A pulse of $-1.7 \mathrm{~V}$ was applied for $0.4 \mathrm{~s}$ just before the deposition stage to form a uniform Ni nucleation on $\mathrm{Si}$, which led to smoother deposition [5]. A metal overlap structure was formed at the edges, suppressing the edge leakage currents. Hence, the formation of a guard ring was not required for these SBs. SBs with Ni layer thicknesses from $200 \mathrm{~nm}$ to $400 \mathrm{~nm}$ were fabricated, but no variation of the SB parameters with thickness was observed.

For comparison, evaporated Ni-Si SBs were also fabricated. Al ohmic back contacts were defined, and Ni-Si Schottky contacts were formed at the front by evaporation and lift-off.
Again, a 20:1 buffered HF dip for $30 \mathrm{~s}$ was used to remove the native oxide and leave the $\mathrm{Si}$ surface $\mathrm{H}$-terminated, just before evaporation.

$I-V$ and $C-V$ characteristics measurements were performed using a Hewlett Packard 4155A semiconductor parameter analyzer and a Hewlett Packard 4280A, $1 \mathrm{MHz}, C$ Meter/C-V plotter. Low temperature $I-V$ measurements down to $86 \mathrm{~K}$ were performed using a Bio-Rad DL 4960 cryostat temperature controller. X-ray diffraction and Scanning Electron Microscopy (SEM) measurements where performed using a Siemens D5000 X-ray Diffractometer and a LEO 1455VP SEM.

\section{Results and discussion}

A typical current density $(J)$ versus applied potential $\left(V_{a}\right)$ characteristic of electrodeposited $\mathrm{Ni}-\mathrm{Si} \mathrm{SBs}$ for $n$ on $n+$ $\mathrm{Si}$ is shown in Fig. 1. The $\mathrm{Si}$ resistivity at the interface is $0.9-1.0 \Omega \mathrm{cm}$. A high-quality rectifying behavior is observed. The $n+$ bulk Si keeps the series resistance low, increasing the exponential region at the forward bias. This allows the extrapolation of the SB parameters with increased accuracy.

For such doping concentrations, tunneling effects can be neglected and therefore thermionic emission is assumed to be the dominant transport mechanism. From the exponential part of the forward bias in Fig. 1, a saturation current density of $J_{S}=1 \mu \mathrm{A} \mathrm{cm}^{-2}$ is extrapolated. Using the Richardson constant value for free electrons $\left(A^{*}=120 \mathrm{~A} \mathrm{~cm}^{-2} \mathrm{~K}^{-2}\right)$, a SB height of $\phi_{B}=0.78 \mathrm{~V}$ is obtained. The reverse leakage matches the saturation current density and has a low field dependence. No size variation of the leakage current and the SB parameters was observed, indicating an effective metal overlap structure at the edges. In higher fields, the junction eventually breaks down. The breakdown typically occurs for reverse biases beyond $10 \mathrm{~V}$.

A typical $J-V$ characteristic of evaporated $\mathrm{Ni}-\mathrm{Si}, \mathrm{SBs}$, with $\mathrm{Si}$ resistivity of $10-20 \Omega \mathrm{cm}$ is also shown in Fig. 1 for comparison. These measurements agree well with others reported in the literature for similar $\mathrm{Ni}-\mathrm{Si}$ contacts [9]. The rectifying behavior is much weaker compared to that of electrodeposited SBs, with a considerably higher reverse bias current. The saturation current density is $1 \mathrm{~mA} \mathrm{~cm}{ }^{-2}$, corresponding to a SB height of $\phi_{B}=0.60 \mathrm{~V}$.

Capacitance $(C)$ versus voltage measurements of SBs on plain $\mathrm{Si}$ were performed for an $A^{*}$-independent measurement of the SB height. An inverse square capacitance versus voltage characteristic is shown in Fig. 2. As expected, a straight line is observed, and from its intercept on the voltage axis the SB height is calculated to be $0.76 \mathrm{~V}$ [10]. Furthermore, from the slope of this characteristic, the $\mathrm{Si}$ doping concentration can be extrapolated. A value of $2.96 \times 10^{15} \mathrm{~cm}^{-3}$ is obtained, corresponding to a resistivity of $1.6 \Omega \mathrm{cm}$, which matches the specification of the Si substrate used $(1-2 \Omega \mathrm{cm})$.

In order to further investigate the quality of $\mathrm{Ni}-\mathrm{Si} \mathrm{SBs}$ obtained by electrodeposition, low temperature $(T) J-V$ measurements were performed. The range was from $86 \mathrm{~K}$ to $320 \mathrm{~K}$, with steps of $10 \mathrm{~K}$. The forward bias characteristics are shown in Fig. 3. For clarity, only 7 different temperatures 


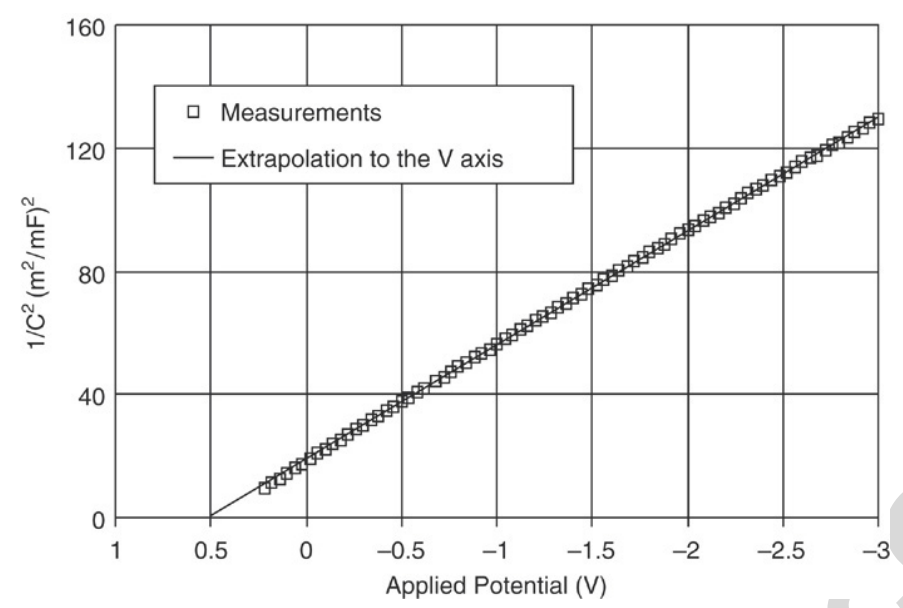

Fig. 2. $C^{-2}-V$ curve of an electrodeposited Ni-Si contact. For the measurements a $1 \mathrm{MHz}$ signal with $30 \mathrm{mV}$ rms was used. From this curve a SB height of $0.76 \mathrm{~V}$ is extrapolated.

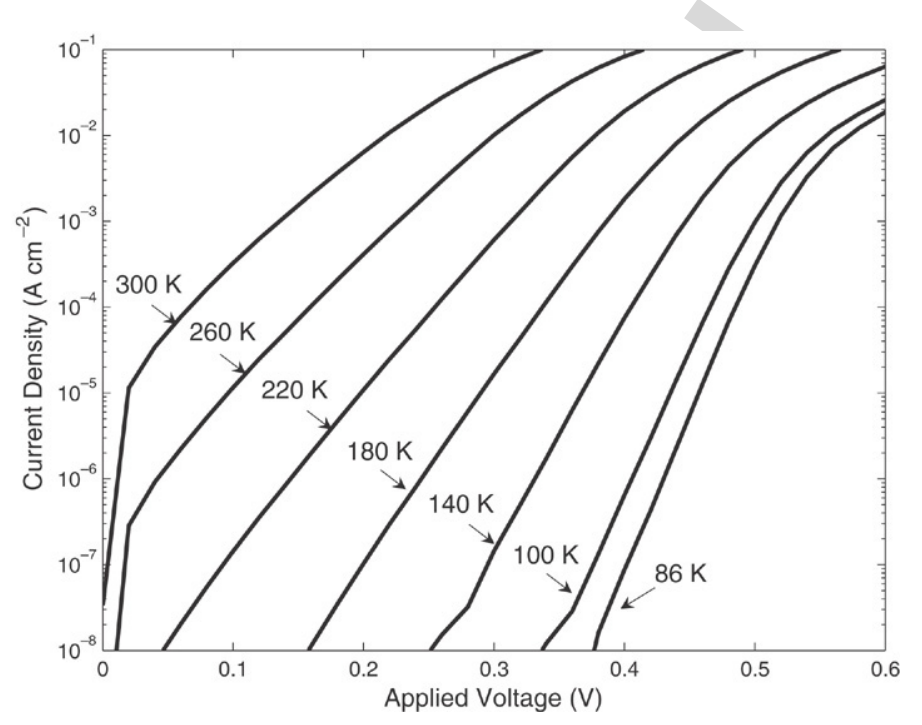

Fig. 3. Low temperature, forward bias $J-V$ characteristics of a circular electrodeposited Ni-Si SB with diameter $0.15 \mathrm{~cm}$.

are plotted in this diagram. As expected from the thermionic emission theory, $J_{S}$ is reduced drastically with temperature. The reverse leakage current is also reduced following $J_{S}$ (not shown in Fig. 3). Below $190 \mathrm{~K}$, the reverse leakage is lower than $1 \mathrm{pA}$, which is the lower current limit of the measurement setup that has been used.

The saturation current density was extrapolated from the $J-V$ measurements for different temperatures, and the activation energy diagram was acquired as shown in Fig. 4. A temperature independent SB height would result in a straight line on the activation energy diagram. A fit with $\phi_{B}=0.70 \mathrm{~V}$ is shown as a solid line in Fig. 4. This line fits only the high temperature experimental results. For lower temperatures, a deviation from a straight line is observed, indicating a temperature dependent SB height.

Several models have been proposed to explain the low temperature behavior of SBs [11]. In order to model the temperature dependence of SB heights, the so-called $T_{0}$ effect is often used [12]. In this model, the thermionic emission equation is modified as:
$J\left(V_{a}\right)=A^{*} \cdot T^{2} \cdot \mathrm{e}^{-\frac{q \cdot \phi_{B n}}{k \cdot\left(T+T_{o}\right)}} \cdot\left(\mathrm{e}^{\frac{q \cdot V_{a}}{k \cdot\left(T+T_{o}\right)}}-1\right)$

where $V_{a}$ is the applied potential, $q$ is the elementary charge, $k$ is the Boltzmann constant, $\phi_{B n}$ is a temperature independent SB height, and $T_{O}$ is a fitting parameter. By using $\phi_{B n}$ and $T_{O}$ as free parameters, a better fit than the temperature independent SB height is achieved (dashed line in Fig. 4). The fitting values were $0.89 \mathrm{~V}$ and $70 \mathrm{~K}$ respectively. For consistency, the same Richardson constant value as for the thermionic emission model was used (120 $\mathrm{A} \mathrm{cm}^{-2} \mathrm{~K}^{-2}$ ). However, this model fails to fit the experimental data for temperatures below $125 \mathrm{~K}$.

A model that physically justifies the temperature dependence of SBs is that proposed by Werner and Guttler [13]. This model assumes a distribution of SB heights as a result of spatial inhomogeneities at the metal/semiconductor interface. The current density is generally given by the following equation:

$J\left(V_{a}\right)=A^{*} \cdot T^{2} \cdot \mathrm{e}^{-\frac{q \cdot \phi_{B n}}{k \cdot T}} \cdot\left(\mathrm{e}^{\frac{q \cdot V_{a}}{k \cdot T}}-1\right)$ 


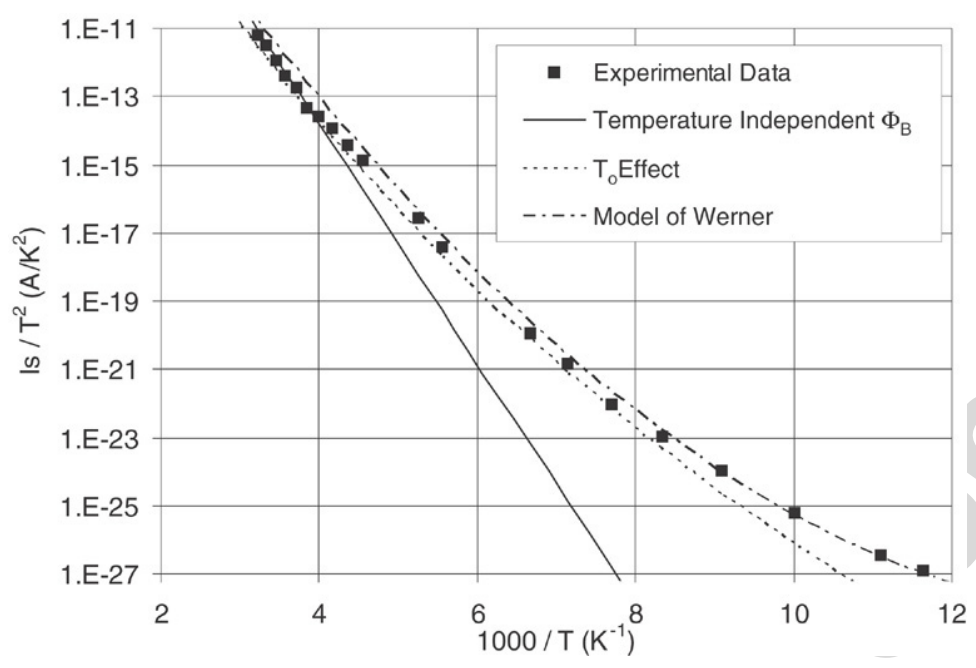

Fig. 4. Activation energy diagram (Arrhenius plot) of the same electrodeposited Ni-Si contact as in Fig. 3. The measurements are fit using a temperature-independent SB height thermionic emission model, the $T_{0}$ effect model [12] and the model of Werner and Guttler [13].

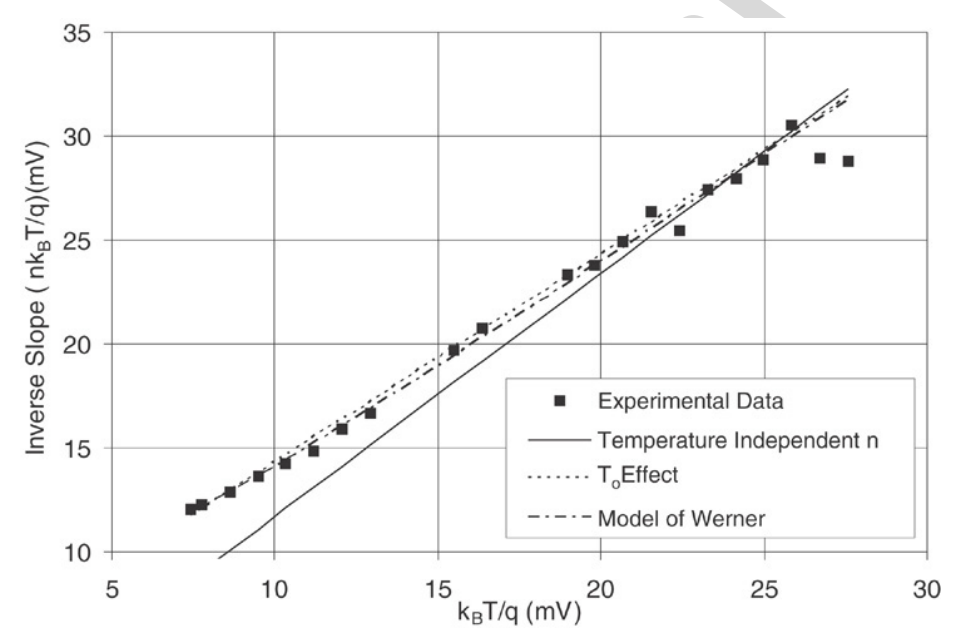

Fig. 5. Inverse slope of the characteristics in Fig. 3 versus $k_{B} T / q$. The measurements are fit using the same models as in Fig. 4 .

where the SB height $\phi_{B n}$ has a temperature dependent distribution:

$\phi_{B n}=\overline{\phi_{B n}}-\frac{q \cdot \sigma_{s}^{2}}{2 \cdot k \cdot T}$

with $\overline{\phi_{B n}}$ and $\sigma_{s}$ being the mean value and the standard deviation of the spatial SB height distribution respectively. If the spatial distribution is on a length scale less than the spacecharge width, then $\overline{\phi_{B n}}$ should match the SB height value obtained by $C-V$ measurements.

In order to fit our experimental results with this model, we use the SB height value from $C-V$ measurements, leaving the standard deviation as the only fit parameter. Again, a value of $120 \mathrm{~A} \mathrm{~cm}^{-2} \mathrm{~K}^{-2}$ for the Richardson constant is used. The resulting fit, using a standard deviation of $66 \mathrm{mV}$, is shown as a dash-dot curve in Fig. 4. An excellent fit is obtained throughout the range of measurements.

To investigate the slope of the $J-V$ characteristics in Fig. 3, the ideality factor $n$ is introduced as:

$J\left(V_{a}\right)=A^{*} \cdot T^{2} \cdot \mathrm{e}^{-\frac{q \cdot \phi_{B}}{k \cdot T}} \cdot\left(\mathrm{e}^{\frac{q \cdot V_{a}}{n \cdot k \cdot T}}-1\right)$.
In this equation, it is assumed that $\phi_{B}$ does not depend on $V_{a}$, and therefore the slope is exclusively defined by the second exponential term.

The inverse slope of the $J-V$ characteristic in Fig. 3 was extrapolated and plotted versus $k T / q$ as shown in Fig. 5. A temperature independent $n$ would result in a zero-crossing straight line, which cannot fit the measurements at low temperatures. A limited fit is achieved at temperatures near $300 \mathrm{~K}$ using an ideality factor of 1.17 (solid line in Fig. 5).

By comparison of Eqs. (1) and (4), one can easily note that the $T_{0}$-effect model corresponds to an ideality factor that varies with temperature as:

$n=1+\frac{T_{0}}{T}$.

This model results in a good fit of the measurements as shown in Fig. 5 (dashed line) with $T_{0}=50 \mathrm{~K}$. However, it lacks a direct physical explanation. Moreover, as implied in Eq. (1), $T_{0}$ should have the same value for the temperature dependence of both $\phi_{B n}$ and $n$. 


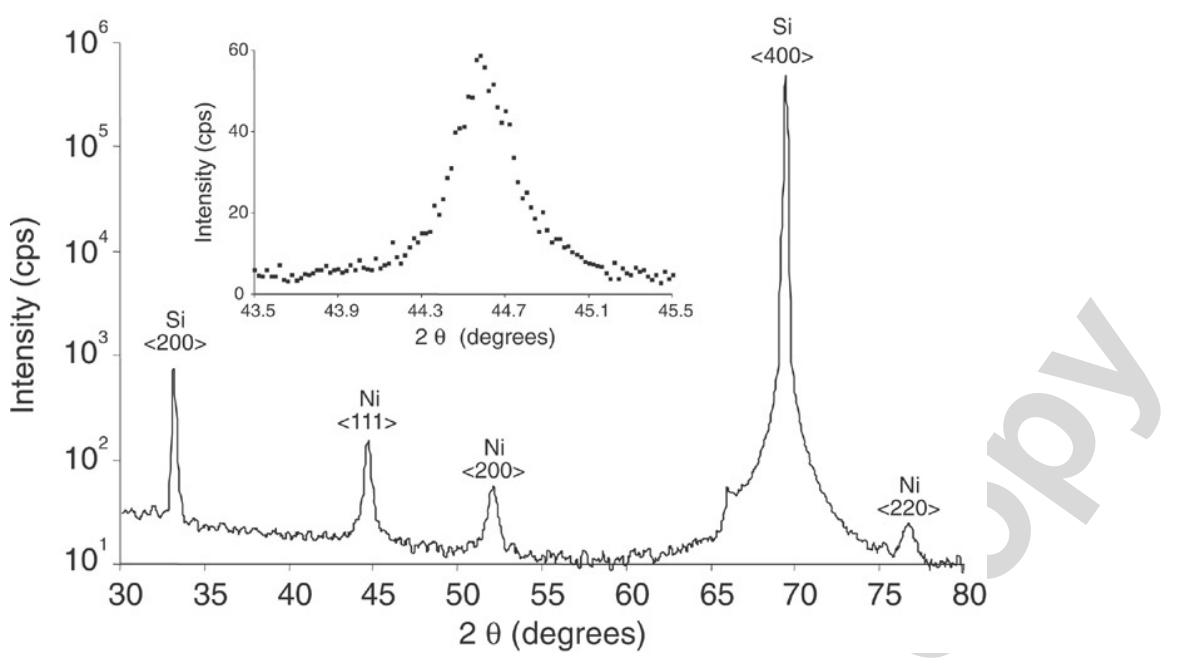

Fig. 6. X-ray diffraction measurements of an electrodeposited Ni-Si SB. The width of the $\langle 111\rangle$ Ni peak (inset) reveals a vertical crystal grain size of 340 nm which is close to the total Ni layer thickness.

The model of Werner and Guttler suggests that the deviation of the ideality factor from unity, and its temperature dependence, are due to the voltage dependence of the barrier distribution [13]. With the assumption that $\overline{\phi_{B n}}$ and $\sigma_{s}^{2}$ vary linearly with bias, with gradients $\rho_{2}$ and $\rho_{3}$ respectively, $n$ will be:

$n=\frac{1}{1-\rho_{2}+\frac{\rho_{3}}{2 k T / q}}$.

A good fit is obtained using $\rho_{2}=0.04$ and $\rho_{3}=-5 \mathrm{mV}$, as shown in Fig. 5 (dash-dot curve). This result, along with the excellent fit of the temperature dependence of the SB height, indicates that the inhomogeneous SB model of Werner and Guttler is an accurate description for electrodeposited SBs.

In order to investigate the physical origin of inhomogeneity at the electrodeposited SBs, X-ray diffraction measurements were performed, and are presented in Fig. 6. The $\langle 400\rangle$ Si peak at $2 \theta=69.7^{\circ}$ and a much lower (forbidden) $\langle 200\rangle \mathrm{Si}$ peak at $33.3^{\circ}$ are observed. These peaks indicate a $\langle 100\rangle$ crystal orientation, as expected from the specifications of the Si substrate. Also, the $\langle 111\rangle,\langle 200\rangle$ and $\langle 220\rangle \mathrm{Ni}$ peaks are observed at $44.66^{\circ}, 51.8^{\circ}$ and $76.3^{\circ}$ respectively. The relative intensities of the Ni peaks are found to be $(\langle 111\rangle:\langle 200\rangle:\langle 220\rangle)=$ (100:36:16), indicating a polycrystalline $\mathrm{Ni}$ layer in good agreement with the relative intensities for polycrystalline $\mathrm{Ni}$ which are 100:42:21 (see [14] and the references therein).

From the width of the $\langle 111\rangle \mathrm{Ni}$ peak shown in the inset of Fig. 6 , the single-crystal height $t$ can be calculated using Scherrer relation: $t=0.9 \lambda /(d \cos \theta)$, where $\lambda$ is the wavelength of the X-rays and $d$ is the full width at halfmaximum measured in radians. The single-crystal height is found to be $340 \mathrm{~nm}$, very close to the thickness of the Ni layer, which was $400 \mathrm{~nm} \pm 10 \%$. This result suggests that the Ni crystal grains span from the $\mathrm{Ni}-\mathrm{Si}$ interface to the top of the $\mathrm{Ni}$ layer.

The formation of such a structure can be explained by studying the growth process of $\mathrm{Ni}$ on $\mathrm{Si}$. For this purpose,

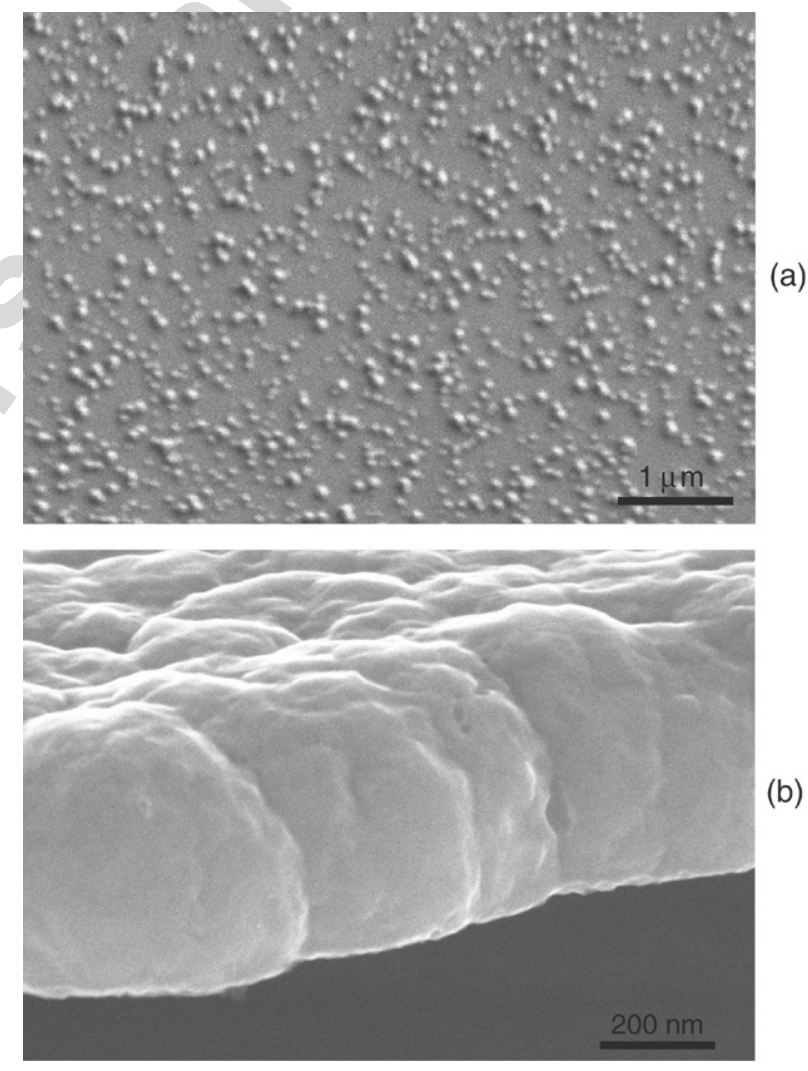

Fig. 7. SEM measurements showing: (a) Nucleation of $\mathrm{Ni}$ on $\mathrm{Si}$ after an electrodeposition pulse of $-1.7 \mathrm{~V}$ for $0.4 \mathrm{~s}$. The gray background is the $\mathrm{Si}$ substrate, while the brighter dots are Ni nucleation sites. (b) Side view of an electrodeposited $\mathrm{Ni}$ film.

SEM measurements were performed during the early stages of electrodeposition. A top-view SEM image of Ni on $\mathrm{Si}$ after an electrodeposition pulse of $-1.7 \mathrm{~V}$ for $0.4 \mathrm{~s}$ is illustrated in Fig. 7(a). The gray background is the $\mathrm{Si}$ substrate, while the brighter dots are $\mathrm{Ni}$ nucleation sites. As these grains grow, they come into contact, and in different areas along the surface, different orientations dominate, resulting ultimately in 
a polycrystalline film. A side-view of an electrodeposited $\mathrm{Ni}$ film is shown in Fig. 7(b).

Different crystal orientations may have different work functions [15]. Therefore, the polycrystalline structure of the Ni layer may result in a spatial distribution of the SB height on a length scale smaller than the depletion width. This could therefore be an explanation for the variation of barrier height that follows from the model of Werner and Gutter.

The above considerations give a possible physical explanation of inhomogeneity at electrodeposited Ni-Si SBs. Further physical characterization by high resolution transmission electron microscopy is required to study the formation of defects and silicides at the electrodeposited $\mathrm{Ni}-\mathrm{Si}$ interface, and to adjudge its viability for spintronic applications.

\section{Conclusion}

Electrodeposited Ni-Si Schottky Barries were characterized by $J-V, C-V$ and low temperature $J-V$ measurements. Electrodeposition was shown to result in higher SB heights compared with evaporation. The reverse leakage current was found to be extremely low, assuring a high quality rectifying behavior. The temperature dependence of the $I-V$ characteristics can be quantitatively fitted by taking into account a model for inhomogeneous SBs proposed by Werner and Guttler. A possible reason for the spatial variation of the SB height is the polycrystalline structure of the Ni layer.

\section{Acknowledgements}

This work was supported by EPSRC, the European Union (SINANO) and Innos Ltd who provided access to clean room processing. We would like to thank M.T. Weller and R.W. Hughes for access to their X-Ray diffractometer.

\section{References}

[1] G. Schmidt, Concepts for spin injection into semiconductors - a review, J. Phys. D: Appl. Phys. 38 (2005) R107-R122.

[2] H. Jaffres, A. Fert, Spin injection from a ferromagnetic metal into a semiconductor, J. Appl. Phys. 91 (2002) 8111-8113.

[3] A.T. Hanbicki, O.M.J. van t Erve, R. Magno, G. Kioseoglou, C.H. Li, B.T. Jonker, G. Itskos, R. Mallory, M. Yasar, A. Petrou, Analysis of the transport process providing spin injection through an Fe/AlGaAs Schottky barrier, Appl. Phys. Lett. 82 (2003) 4092-4094.

[4] A.M. Bratkovsky, V.V. Osipov, Efficient spin injection and extraction in modified reverse and forward biased ferromagnetic-semiconductor junctions and low-power ultrafast spin injection devices, Appl. Phys. A: Mater. Sci. Proc. 80 (2005) 1237-1246.

[5] M.E. Kiziroglou, A.A. Zhukov, M. Abdelsalam, X. Li, P.A.J. de Groot, P.N. Bartlett, C.H. de Groot, Electrodeposition of Ni-Si Schottky barriers, IEEE Trans. Magn. 41 (10) (2005) 2639-2641.

[6] X. Zhang, E. Garfunkel, Y.J. Chabal, S.B. Christman, E.E. Chaban, Stability of HF-etched S(100) surfaces in oxygen ambient, Appl. Phys. Lett. 79 (24) (2001) 4051-4053.

[7] M. Yoshimura, I. Ono, K. Ueda, Initial stages of Ni reaction on Si (100) and $H$-terminated Si (100) surfaces, Appl. Surf. Sci. 130-132 (1998) 276-281

[8] P.N. Bartlett, M.A. Ghanem, I.S.E. Hallag, P. de Groot, A. Zhukov, Electrochemical deposition of macroporous magnetic networks using colloidal templates, J. Mater. Chem. 13 (2003) 2596-2602.

[9] J.M. Shannon, Reducing the effective height of a Schottky barrier using low-energy ion implantation, Appl. Phys. Lett. 24 (8) (1974) 369-371.

[10] S.M. Sze, Physics of Semiconductor Devices, Wiley Interscience, 1981.

[11] R.T. Tung, Electron Transport at metal-semiconductor interfaces: General theory, Phys. Rev. B 45 (23) (1992) 13509-13523.

[12] A.N. Saxena, Forward current-voltage characteristics of Schottky barriers on n-type silicon, Surf. Sci. 13 (1969) 151-171.

[13] J.H. Werner, H.H. Guttler, Barrier inhomogeneities at Schottky contacts, J. Appl. Phys. 69 (3) (1991) 1522-1533.

[14] A.G. McCormack, M.J. Pomeroy, V.J. Cunnane, Microstructural development and surface characterization of electrodeposited Nickel/Yttria composite coatings, J. Electrochem. Comm. 150 (5) (2003) C356-C361.

[15] H.B. Michaelson, The work function of the elements and its periodicity, J. Appl. Phys. 48 (11) (1977) 4729-4733. 